\title{
Insulin-like growth factor-1 rescues synaptic and motor deficits in a mouse model of autism and developmental delay
}

\author{
Ozlem Bozdagi $i^{1,2}$, Teresa Tavassoli ${ }^{1,2}$ and Joseph D Buxbaum ${ }^{1,2,3,4,5,6^{*}}$
}

\begin{abstract}
Background: Haploinsufficiency of SHANK3, due to either hemizygous gene deletion (termed 22q13 deletion syndrome or Phelan-McDermid syndrome) or to gene mutation, accounts for about $0.5 \%$ of the cases of autism spectrum disorder (ASD) and/or developmental delay, and there is evidence for a wider role for SHANK3 and glutamate signaling abnormalities in ASD and related conditions. Therapeutic approaches that reverse deficits in SHANK3-haploinsufficiency may therefore be broadly beneficial in ASD and in developmental delay.

Findings: We observed that daily intraperitoneal injections of human insulin-like growth factor 1 (IGF-1) over a 2-week period reversed deficits in hippocampal a-amino-3-hydroxy-5-methyl-4-isoxazolepropionic acid (AMPA) signaling, long-term potentiation (LTP), and motor performance that we had previously reported in Shank3-deficient mice. Positive effects were observed with an IGF-1 peptide derivative as well.

Conclusions: We observed significant beneficial effects of IGF-1 in a mouse model of ASD and of developmental delay. Studies in mouse and human neuronal models of Rett syndrome also show benefits with IGF-1, raising the possibility that this compound may have benefits broadly in ASD and related conditions, even with differing molecular etiology. Given the extensive safety data for IGF-1 in children with short stature due to primary IGF-1 deficiency, IGF-1 is an attractive candidate for controlled clinical trials in SHANK3-deficiency and in ASD.
\end{abstract}

Keywords: Pharmacotherapy, Personalized medicine, Individualized medicine, 22q13 deletion syndrome, Phelan-McDermid syndrome

\section{Findings}

SHANK proteins are master scaffolding proteins of the postsynaptic density (PSD) of glutamatergic synapses and are critical determinants of glutamate transmission and synaptic spine dynamics [1]. Loss of one functional copy of SHANK3 accounts for about $0.5 \%$ of the cases of autism spectrum disorder (ASD) and/or developmental delay [2], and there is likely a wider role for SHANK3 and glutamate signaling abnormalities in ASD and related neurodevelopmental disorders [3,4]. Targeted disruption of the full-length form of Shank3 (sometimes called Shank3a) in mice leads to deficits in hippocampal

\footnotetext{
* Correspondence: joseph.buxbaum@mssm.edu

'Seaver Autism Center for Research and Treatment, Icahn School of Medicine at Mount Sinai, New York, NY, USA

${ }^{2}$ Department of Psychiatry, Icahn School of Medicine at Mount Sinai, New York, NY, USA

Full list of author information is available at the end of the article
}

AMPA signaling, long-term potentiation (LTP), and motor performance [5-7], likely reflecting delayed synaptic development as shown by the reduced AMPA signaling [5] and decreased levels of PSD-95 (unpublished results). IGF-1, which enters the central nervous system (CNS) through an interaction with lipoprotein-related receptor 1 (LRP1) [8], has multiple effects on neuronal and synaptic development and function, including effects on neurogenesis and synaptogenesis [9]. IGF-1 treatment also enhances the PSD as measured both by PSD length and by levels of PSD-95 [10,11]. Recombinant human IGF-1 has substantial human safety data and is approved for use in children, making IGF-1 an attractive compound for evaluation in neurodevelopmental disorders.

To investigate whether IGF-1 could reverse deficits in a preclinical model of SHANK3-haploinsufficiency, we made use of a mouse with hemizygous loss of full-length Shank3 due to targeted disruption of the ankyrin repeat 
domain (ARD) [5]. This isoform has been directly implicated in ASD, language delay, and intellectual disability (ID), as there exist disruptive de novo point mutations in ARD in patients with ASD and ID [12,13]. In all studies, we compared heterozygous mice with wild-type littermates using heterozygote $\times$ heterozygote mating. Consistent with previous results from our group [5], LTP induced by high-frequency stimulation was reduced in the heterozygous mice compared to wild-type littermates in the current experiments (Figures 1a and 2a) (for
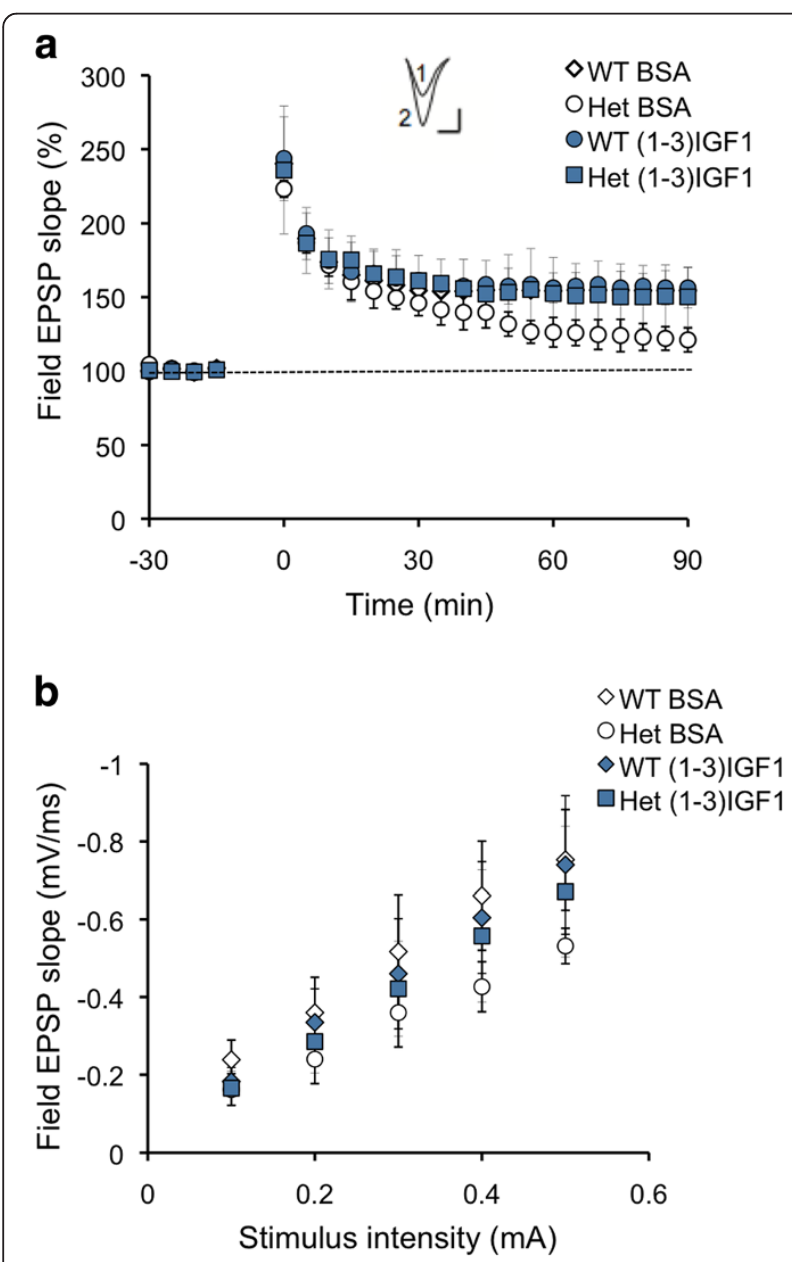

Figure 1 (1-3)IGF-1 reverses deficits in LTP and basal synaptic properties in Shank3-deficient mice. Wild-type (WT) and heterozygous (Het) mice were treated with saline or (1-3)|GF-1 for 2 weeks before testing (injections began at postnatal day (PND) 13 to 15 and animals were analyzed immediately after the last injection). Methods for all experiments were as described previously [5], with 3 to 4 mice per group, and 1 to 2 slices per animal. (a) Hippocampal LTP was induced with high-frequency stimulation. Inset: Representative excitatory postsynaptic potential traces at 90 min after LTP induction from saline-injected (1) and (1-3)|GF-1-injected (2) heterozygous mice (scale bar: 0.5 mV, 10 ms). (b) Input-output curves comparing field excitatory postsynaptic potential (EPSP) slopes ( $\mathrm{mV} / \mathrm{ms}$ ) as a function of stimulation intensity (mA). EPSP: excitatory postsynaptic potential; Het: heterozygous; LTP: long-term potentiation; PND: postnatal day; WT: wild-type. example, in Figure 2a, repeated measures ANOVA was used for analysis of the last five time points, $F(1,6)=$ 33.71, $P=0.001$ ).

We first tested an active peptide derivative of IGF-1, (1-3)IGF-1, which has been shown to cross the bloodbrain barrier and rescue Rett syndrome symptoms in Mecp2-deficient mice [11]. We observed that intraperitoneal injections at $10 \mu \mathrm{g} / \mathrm{g} /$ day for 2 weeks restored normal hippocampal LTP in Shank3 heterozygous mice but had no effect on wild-type mice (repeated measures ANOVA was used to analyze the last five time points, $F(3,11)=6.07, P=0.011)$. In post hoc analyses, vehicletreated heterozygous mice were significantly different from wild-type mice $(P=0.004)$, while $(1-3)$ IGF-1 treated heterozygous mice were not $(P=0.66)$. Furthermore, peptide treatment reversed deficits in the mean slope of the input/ output (I/O) function (Figure 1b) (one-way ANOVA, $F(3,19)=4.25, P=0.02)$. Vehicle-treated heterozygous mice were significantly different from vehicle-treated wild-type mice $(P=0.001)$, while $(1-3)$ IGF-1 treated heterozygous mice were not different from vehicletreated wild-type $(P=0.89)$, and there were no significant differences between vehicle-treated wild-type mice and wild-type mice treated with IGF-1 $(P=0.812)$, so further studies used just three conditions.

We next administered full-length IGF-1, like that used in children with short stature due to primary IGF-1 deficiency, by intraperitoneal injection at $240 \mu \mathrm{g} / \mathrm{kg} / \mathrm{day}$, starting at PND 13 to 15 and continuing for 2 weeks (Figure 2a). This dose, chosen because it represents the maximum dose according to the current FDA label for IGF-1, was effective in rescuing deficits in LTP (repeated measures ANOVA was used to analyze the last five time points, comparing heterozygous mice with and without IGF-1, $F(1,6)=28.04, P=0.002)$. In contrast, lower dose IGF$1(120 \mu \mathrm{g} / \mathrm{kg} /$ day for 2 weeks) was associated with more modest reversal of deficits in LTP (for the last five time points: $F(1,6)=2.62, P=0.012$ ), showing a dose-response effect and providing preclinical dosing information.

Specific deficits in the glutamate AMPA receptor component of neural signaling [5] were also reversed by a 2-week treatment of $240 \mu \mathrm{g} / \mathrm{kg} /$ day full-length IGF-1 (Figure $2 \mathrm{~b}$ ). The mean slope of the I/O function was $0.50 \pm 0.14$ for wild-type, $0.34 \pm 0.06$ for Shank3 heterozygotes and $0.61 \pm$ 0.059 for IGF-1 injected heterozygotes (one-way ANOVA, $F(2,9)=8.62, P=0.008)$. In post hoc analyses, vehicletreated heterozygous mice were significantly different from vehicle-treated wild-type mice $(P=0.039)$, while IGF-1-treated heterozygous mice were not different from vehicle-treated wild-type mice $(P=0.12)$.

Patients with SHANK3-haploinsufficiency frequently present with hypotonia and motor deficits of variable severity, and we have observed subtle motor deficits in Shank3-heterozygous mice [5,7]. After treating male 

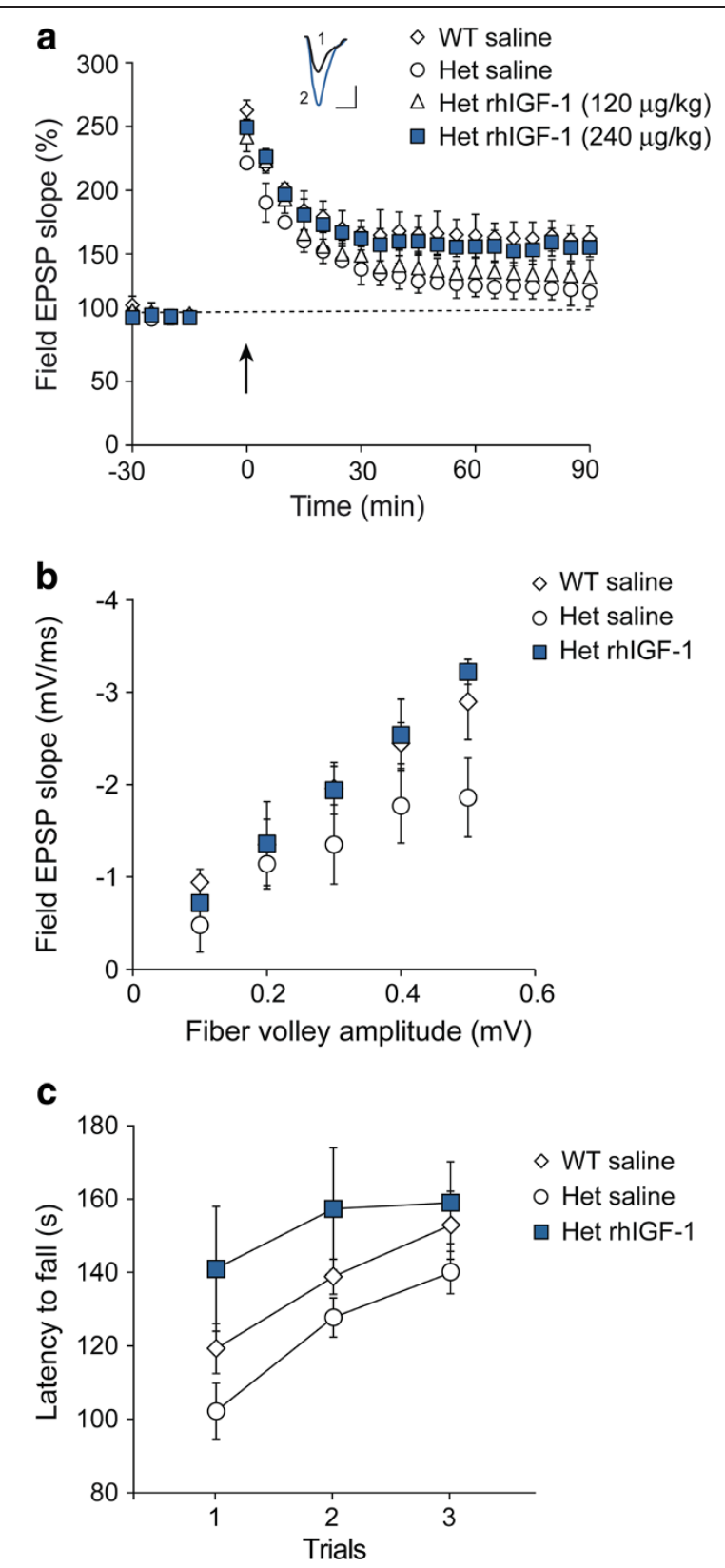

Figure 2 IGF-1 reverses deficits in LTP, AMPA signaling, and motor function in Shank3-deficient mice. Wild-type (WT) and heterozygous (Het) mice were treated with saline or recombinant human IGF-1 (rhIGF-1) for 2 weeks (beginning at PND 13 to 15) before testing and analyzed immediately after the last injection. Methods for all experiments were as described previously [5,7], with 4 to 9 mice per group. (a) Hippocampal LTP was induced with high-frequency stimulation. Inset: Representative excitatory postsynaptic potential traces at 90 min after LTP induction from saline-injected (1) and rhlGF-1 -injected (2) heterozygous mice (scale bar: $0.5 \mathrm{mV}, 10 \mathrm{~ms}$ ). (b) Slices were incubated in the presence of the N-Methyl-D-aspartate (NMDA) antagonist R-2-amino-5-phosphonopentanoate (APV) to expose AMPA receptor signaling. (c) Mice were tested for motor performance and motor learning by measuring latencies to fall off a rotating rod over three trials. Het: heterozygous; LTP: long-term potentiation; NMDA: NMethyl-D-aspartate; rhIGF-1: recombinant human IGF-1; WT: wild-type. heterozygous mice with $240 \mu \mathrm{g} / \mathrm{kg} /$ day for 2 weeks, we observed enhanced motor performance following treatment (Figure 2c) $(F(2,20)=3.98, P=0.03)$.

Our results provide preclinical evidence for a beneficial role for IGF-1 in SHANK3-haploinsufficiency. Moreover, as there is emerging evidence that the SHANK3 pathway and the postsynaptic density, which it helps sculpt, play a role in many neurodevelopmental disorders, as evidenced by large-scale genetic, proteomic, and gene expression studies $[3,4,14]$, therapies for SHANK3 deficiency and synaptic development represent important targets that could have a widespread positive impact for neurodevelopmental disorders. The beneficial effects of IGF-1 in models of Rett syndrome $[11,15]$ are consistent with this hypothesis.

There are some limitations to the current study. We, and others working with similar Shank3-deficient mice, see only limited behavioral abnormalities, with none except for rotarod deficits at the ages where we carried out the IGF-1 treatments and electrophysiological studies. For this reason, the phenotypes we measure are somewhat limited. In addition, a mechanistic understanding of the neuronal effects of IGF-1 has eluded the neuroscience community and we cannot precisely explain how IGF-1 reverses the deficits observed. We do hope, however, that our findings, together with those on IGF-1 in Rett syndrome models, may help spur further research on the action of IGF-1 in the CNS. We did not see any effect produced by the (1-3)IGF-1 peptide on control animals but we did not test the effects of full-length IGF-1 on wild-type mice. There could be enhanced LTP or rotarod performance in control animals following treatment with full-length IGF-1. Many drugs have effects on both healthy and non-healthy individuals and there is hence no a priori reason to assume that IGF-1 has no effect on control animals. In fact, given the positive effects of IGF-1 in Rett syndrome models it is likely that IGF-1 has a general effect on CNS function, which might also be observed in controls.

In summary, our results show that IGF-1, approved for use in children, can lead to functional improvements in a mouse model of ASD and developmental delay, representing an important preclinical step towards novel therapeutics. Clinical trials of IGF-1 in SHANK3deficient individuals and in ASD are now underway (ClinicalTrials.gov Identifier NCT01525901).

\section{Abbreviations}

ARD: Ankyrin repeat domain; ASD: Autism spectrum disorder; AMPA: a-Amino-3-hydroxy-5-methyl-4-isoxazolepropionic acid; CNS: Central nervous system; EPSP: Excitatory postsynaptic potential; Het: Heterozygous; ID: Intellectual disability; IGF-1: Insulin-like growth factor 1; I/O: Input/output; LRP1: Lipoprotein-related receptor 1; LTP: Long-term potentiation; NMDA: N-Methyl-D-aspartate; PND: Postnatal day; PSD: Postsynaptic density; rhIGF-1: Recombinant human IGF-1; WT: Wild-type. 


\section{Competing interests}

$\mathrm{OB}$ and JDB have submitted a patent on this work.

\section{Authors' contributions}

$\mathrm{OB}$ and JDB designed the experiments, interpreted the results, and prepared the manuscript. OB carried out all electrophysiological and behavioral studies. $\Pi$ analyzed the results and help in aspects of experimental design. All authors read and approved the final manuscript.

\section{Acknowledgements}

This work was supported by the Seaver Foundation, the Simons Foundation, the NIMH (grant R01MH093725 to JDB), and by a gift from William Gibson and Paulina Rychenkova, PhD. We thank Catalina Betancur for helpful comments. Aspects of this work were presented at annual meetings of The Society for Neuroscience $(2010,2011)$ and the American Society of Human Genetics (2010), and at Phelan-McDermid Syndrome symposia (2011, 2012).

\section{Author details}

'Seaver Autism Center for Research and Treatment, Icahn School of Medicine at Mount Sinai, New York, NY, USA. ²Department of Psychiatry, Icahn School of Medicine at Mount Sinai, New York, NY, USA. ${ }^{3}$ Department of Neuroscience, Icahn School of Medicine at Mount Sinai, New York, NY, USA. ${ }^{4}$ Department of Genetics and Genomics Sciences, Icahn School of Medicine at Mount Sinai, New York, NY, USA. ${ }^{5}$ Friedman Brain Institute, Icahn School of Medicine at Mount Sinai, New York, NY, USA. ${ }^{6}$ Mindich Child Health and Development Institute, Icahn School of Medicine at Mount Sinai, New York, NY, USA.

Received: 2 January 2013 Accepted: 16 April 2013

Published: 27 April 2013

\section{References}

1. Boeckers TM, Bockmann J, Kreutz MR, Gundelfinger ED: ProSAP/Shank proteins - a family of higher order organizing molecules of the postsynaptic density with an emerging role in human neurological disease. J Neurochem 2002, 81(5):903-910.

2. Abrahams BS, Geschwind DH: Advances in autism genetics: on the threshold of a new neurobiology. Nat Rev Genet 2008, 9(5):341-355.

3. Sakai Y, Shaw CA, Dawson BC, Dugas DV, Al-Mohtaseb Z, Hill DE, et al: Protein interactome reveals converging molecular pathways among autism disorders. Sci Transl Med 2011, 3(86):86ra49.

4. Darnell JC, Van Driesche SJ, Zhang C, Hung KY, Mele A, Fraser CE, et al: FMRP stalls ribosomal translocation on mRNAs linked to synaptic function and autism. Cell 2011, 146(2):247-261.

5. Bozdagi O, Sakurai T, Papapetrou D, Wang X, Dickstein DL, Takahashi N, et al: Haploinsufficiency of the autism-associated Shank3 gene leads to deficits in synaptic function, social interaction, and social communication. Mol Aut 2010, 1(1):15.

6. Wang X, McCoy PA, Rodriguiz RM, Pan Y, Je HS, Roberts AC, et al: Synaptic dysfunction and abnormal behaviors in mice lacking major isoforms of Shank3. Hum Mol Genet 2011, 20(15):3093-3108.

7. Yang M, Bozdagi O, Scattoni ML, Wohr M, Roullet Fl, Katz AM, et al: Reduced excitatory neurotransmission and mild autism-relevant phenotypes in adolescent Shank3 null mutant mice. J Neurosci 2012, 32(19):6525-6541.

8. Nishijima T, Piriz J, Duflot S, Fernandez AM, Gaitan G, Gomez-Pinedo U, et al: Neuronal activity drives localized blood-brain-barrier transport of serum insulin-like growth factor-I into the CNS. Neuron 2010, 67(5):834-846.

9. O'Kusky JR, Ye P, D'Ercole AJ: Insulin-like growth factor-I promotes neurogenesis and synaptogenesis in the hippocampal dentate gyrus during postnatal development. J Neurosci 2000, 20(22):8435-8442.

10. Shi L, Linville MC, Tucker EW, Sonntag WE, Brunso-Bechtold JK: Differential effects of aging and insulin-like growth factor-1 on synapses in CA1 of rat hippocampus. Cereb Cortex 2005, 15(5):571-577.

11. Tropea D, Giacometti E, Wilson NR, Beard C, McCurry C, Fu DD, et al: Partial reversal of Rett syndrome-like symptoms in MeCP2 mutant mice. Proc Natl Acad Sci USA 2009, 106(6):2029-2034

12. Moessner R, Marshall CR, Sutcliffe JS, Skaug J, Pinto D, Vincent J, et al: Contribution of SHANK3 mutations to autism spectrum disorder. Am J Hum Genet 2007, 81(6):1289-1297.
13. Hamdan FF, Gauthier J, Araki Y, Lin DT, Yoshizawa Y, Higashi K, et al: Excess of de novo deleterious mutations in genes associated with glutamatergic systems in nonsyndromic intellectual disability. Am J Hum Genet 2011, 88(3):306-316.

14. Laumonnier F, Cuthbert PC, Grant SG: The role of neuronal complexes in human X-linked brain diseases. Am J Hum Genet 2007, 80(2):205-220.

15. Marchetto MC, Carromeu C, Acab A, Yu D, Yeo GW, Mu Y, et al: A model for neural development and treatment of Rett syndrome using human induced pluripotent stem cells. Cell 2010, 143(4):527-539.

doi:10.1186/2040-2392-4-9

Cite this article as: Bozdagi et al:: Insulin-like growth factor-1 rescues synaptic and motor deficits in a mouse model of autism and developmental delay. Molecular Autism 2013 4:9.

\section{Submit your next manuscript to BioMed Central and take full advantage of:}

- Convenient online submission

- Thorough peer review

- No space constraints or color figure charges

- Immediate publication on acceptance

- Inclusion in PubMed, CAS, Scopus and Google Scholar

- Research which is freely available for redistribution

Submit your manuscript at www.biomedcentral.com/submit
C Biomed Central 